\title{
Método para Segmentação Automática de Lesões de Esclerose Múltipla em exames FLAIR
}

\author{
Pedro Costa Klein ${ }^{1,3}$, Alexandre Rosa Franco ${ }^{2}$, Márcio Sarroglia Pinho ${ }^{1,3}$ \\ ${ }^{1}$ Faculdade de Informática, ${ }^{2}$ Faculdade de Engenharia - Pontifícia Universidade \\ Católica do Rio Grande do Sul (PUCRS) \\ Av. Ipiranga, 6681 - 90.619-900 - Porto Alegre - RS - Brazil \\ ${ }^{3}$ Instituto Nacional de Ciência e Tecnologia em Medicina Assistida por Computação \\ Científica (INCT/MACC) \\ pedro.kleineacad.pucrs.br, \{pinho, alexandre.franco\}@pucrs.br
}

\begin{abstract}
This paper proposes a method for automatic Multiple Sclerosis lesion segmentation in Fluid Attenuated Inverse Recovery (FLAIR) Magnetic Ressonance Images. Unlike the current golden standard method, which requires that in addition to the FLAIR images the $T 1$ images must also be acquired, the approach here described intends to replace the usage of $T 1$ images, used on the determination of the brain structures, by the usage of probabilistic atlases used for the same purpose. As preliminary results, it was obtained a segmentation very close to the golden standard, which for this work in progress indicates a good study direction.
\end{abstract}

Resumo. O presente artigo propõe um método para segmentação automática de lesões de Esclerose Múltipla em imagens de Ressonância Magnética obtidas na modalidade Fluid Attenuated Inverse Recovery (FLAIR). Ao contrário do método considerado padrão ouro atual, que exige que além das imagens em FLAIR também sejam adquiridas imagens na modalidade T1, a abordagem aqui descrita visa substituir o uso das imagens T1, utilizadas na delimitação das estruturas cerebrais, pelo uso de atlas probabilísticos utilizados para os mesmos fins. Como resultados preliminares, obteve-se uma segmentação bastante aproximada do método considerado padrão ouro, o que para este trabalho em andamento indica um bom direcionamento de estudos.

\section{Introdução}

Esclerose Múltipla (EM) é uma doença inflamatória autoimune que atinge o cérebro e a medula espinhal. A doença caracteriza-se pelo ataque do sistema imunológico à mielina presente nas estruturas responsáveis pela condução de pulsos elétricos, o que resulta em lesões na região de substância branca, observáveis como regiões hiperintensas em imagens de ressonância magnética (IRMs) convencionais do tipo T2 ou FLAIR. Esta característica torna o uso de IRMs a ferramenta mais comum para o diagnóstico manual da EM.

Apesar de ser o padrão na prática clínica, a análise manual fica restrita à avaliação da existência ou não de lesões. Quando é preciso segmentar e quantificar tais lesões e acompanhar sua evolução, a execução manual do procedimento se torna demorada e por vezes inviável de ser realizada em larga escala. Além disto, o diagnóstico manual pode sofrer de variâncias entre segmentações realizadas por diferentes profissionais em um mesmo paciente ou até mesmo em segmentações realizadas por um mesmo profissional, em um mesmo paciente ao longo do tempo. 
Assim, o desenvolvimento de métodos automáticos mostra-se necessário para reduzir interações humanas, bem como a variabilidade correspondente a estas interações e permitir a segmentação eficiente de diversas IRMs (GARCÍA-LORENZO et al., 2013).

Nos principais métodos de segmentação/quantificação automática de lesões de EM (GARCÍA-LORENZO et al., 2013), observa-se a necessidade de aquisição de sequências de IRM em duas modalidades: uma em T1 e outra em T2 ou FLAIR. As sequências de IRMs obtidas em T1 apresentam um melhor contraste entre os tecidos cerebrais e, no caso da segmentação automática, servem de base para a identificação dos tecidos cerebrais durante o pré-processamento das imagens. Já as imagens FLAIR apresentam hiperintensidades nas regiões onde ocorrem lesões de substância branca.

A necessidade de múltiplos tipos de IRMs acaba por reduzir a aplicabilidade destes métodos, uma vez que na prática clinica não existe a necessidade de se trabalhar com a modalidade T1. Assim, a adoção de um método automatizado gera a necessidade de uma nova modalidade de IRM ao escopo, o que acarreta em novos custos e uma incapacidade de reprodução do método em exames antigos.

Tendo isto em mente, este trabalho propõe a criação de um método de segmentação automática que utilize apenas imagens FLAIR, já usadas na prática clínica de diagnóstico de EM. A modelagem inicial do método e os resultados obtidos até o momento são apresentados no restante deste artigo.

\section{Método Proposto}

Ao se descartar os exames em T1, surge a necessidade de delimitar os tecidos cerebrais de outra forma. Para tal, propõe-se a utilização de atlas de estruturas cerebrais, como o gerado pelo International Consortium for Brain Mapping (ICBM), ajustados às imagens em que se pretende realizar a segmentação/quantificação das lesões.

Para aplicação do método proposto, foram utilizadas imagens de 10 pacientes fornecidas por um projeto de pesquisa no qual foi adquirido imagens nas modalidades T1 e FLAIR. Em conjunto, foi utilizado o template ICBM 2009a Nonlinear Symmetric (MCCONNEL BRAIN IMAGING CENTRE, 1997). Deste template foram utilizadas as imagens ponderadas em T1 e as máscaras de substância branca.

De forma geral, o método proposto consiste no registro da imagem do atlas para o espaço da IRM do paciente, na segmentação da região de substância branca nos exames do paciente (a partir do atlas registrado) e na identificação de zonas hiperintensas nesta região, uma vez que comportamentos de hiperintensidade são característicos de zonas de lesão em imagens do tipo FLAIR (GARCÍA-LORENZO et al., 2013).

O algoritmo proposto, descrito na Figura 1, inicia registrando as imagens do template ICBM para o espaço dos exames do paciente, garantindo assim que os voxels nos templates e no exame correspondam à mesma localização no espaço cerebral. permite a identificação e a extração da região de substância branca no exame FLAIR.

Uma vez extraídas as regiões de substância branca, aplica-se uma binarização por limiar de forma a identificar as regiões de hiperintensidade presentes neste tecido, o que por consequência evidencia regiões de lesão de substância branca. 


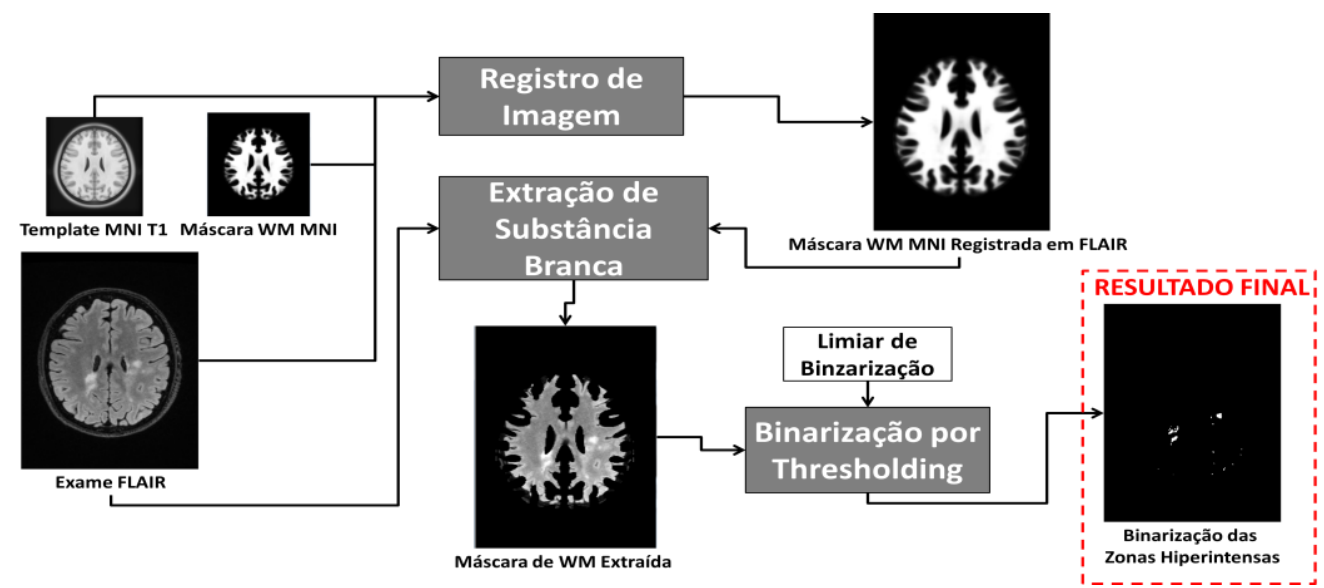

Figura 1 - Pipeline de Execução do Método Proposto

\section{Resultados Preliminares}

A fim de avaliar os resultados obtidos através da aplicação deste método, foi realizada uma comparação dos resultados preliminares deste método com os resultados obtidos através da aplicação do método utilizado pelo Lesion Segmentation Tool (LST) (SCHMIDT et al., 2012), considerado atualmente como o padrão ouro em segmentação automática de lesões de EM. É importante ressaltar que a utilização desta ferramenta só foi possível porque os exames fornecidos pelo projeto possuíam também aquisições na modalidade $\mathrm{T} 1$.

A fim de se trabalhar com o mesmo espaço para as duas abordagens, foi aplicado o pipeline de execução do método proposto nas imagens pré-processadas no LST. A etapa de pré-processamento do LST corrige inomogeneidades e registra os exames FLAIR no espaço T1.

Do ponto de vista qualitativo, observa-se, na Figura 2, que os resultados obtidos através da limiarização das regiões de substância branca aproximaram-se significativamente do resultado obtido com o padrão ouro. Os resultados obtidos através do LST são apresentados na região superior da imagem, em verde; e os resultados obtidos através do método proposto, na região inferior da imagem, em vermelho.

Do ponto de vista quantitativo, entretanto, percebeu-se uma diferença significativa no cálculo da volumetria das lesões. Através do uso do módulo "Compute total lesion volume" do LST, que recebe como entrada imagens binárias das lesões, obtém-se uma quantificação de $9,381 \mathrm{ml}$ para o LST, contra 4,406ml para o método proposto.

\section{Conclusão e próximos passos}

Mesmo em face às limitações observadas nos resultados do método proposto, pode-se notar que apenas a aplicação da limiarização, quando bem direcionada (regiões de substância branca), surte um efeito significativamente próximo do que se encontra no estado da arte.

Além disto, a eliminação da obrigatoriedade de aquisição de mais uma modalidade (T1) expande o escopo de aplicação da segmentação automática, permitindo sua utilização tanto em exames já realizados quanto em exames novos, facilitando o acompanhamento da progressão da doença. 
Resta como tarefa para a este trabalho a implementação do pré-processamento das imagens, semelhante ao que propõe o padrão ouro; o refinamento do limiar de binarização; e algum pós-tratamento nas lesões encontradas, de forma a buscar-se que os clusters de voxels hiperintensos possuam o tamanho semelhante ao apresentado no padrão ouro.
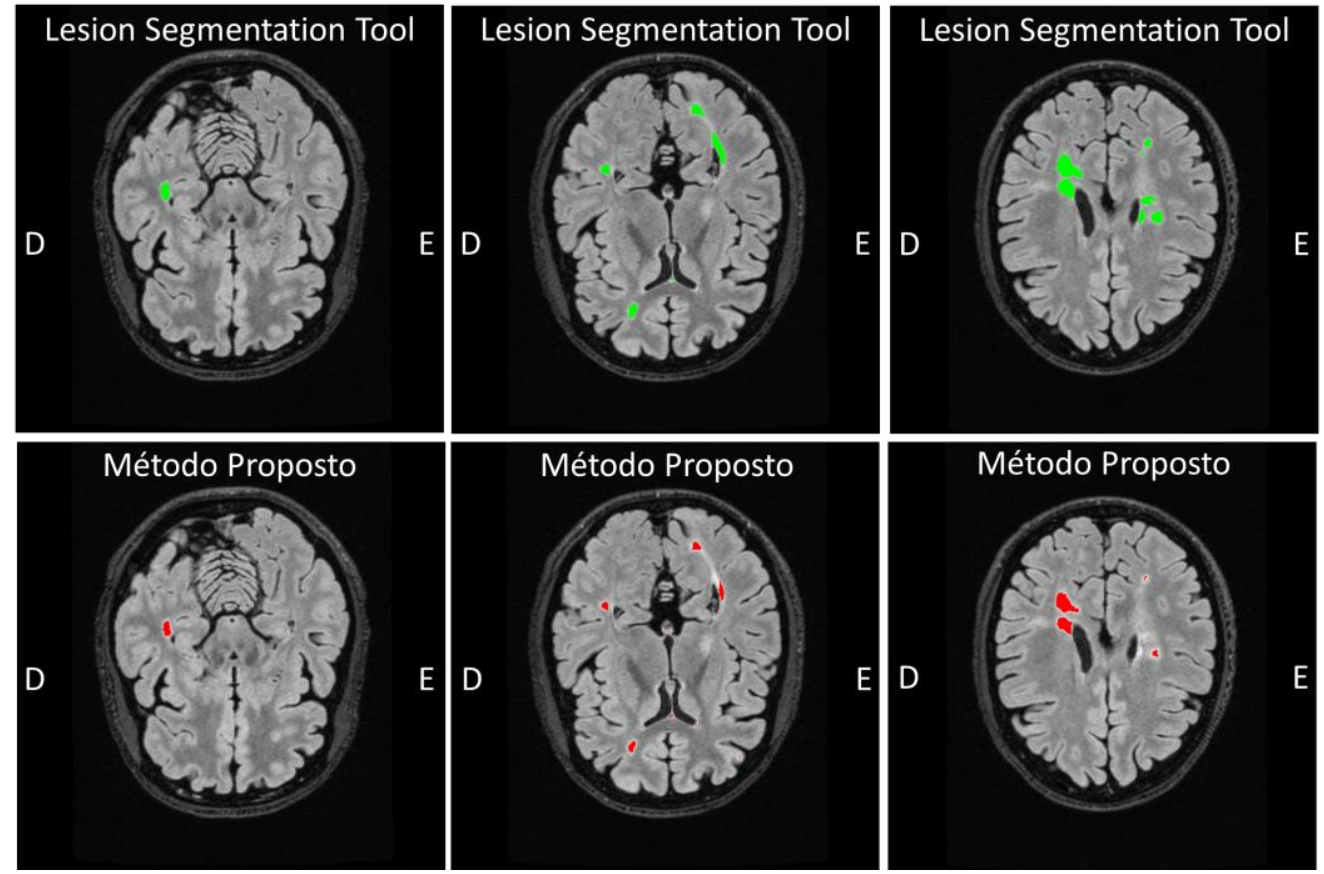

Figura 2 - Comparativo visual entre os resultados obtidos pela LST (superior em verde) e pelo uso do método proposto (inferior em vermelho), sobrepostos ao exame FLAIR.

\section{Agradecimentos}

Este pesquisa foi parcialmente financiada pelo Instituto Nacional de Ciência e Tecnologia em Medicina Assistida por Computação Científica. (CNPq 181813/2010-6 and FAPERJ E- 26/170.030/2008).

\section{Referências}

GARCÍA-LORENZO，D.; FRANCIS，S.; NARAYANAN， S.; ARNOLD，D. L.; COLLINS, D. L. Review of automatic segmentation methods of multiple sclerosis white matter lesions on conventional magneti resonance imaging. Medical Image Analysis, 2013. 1-18.

MCCONNEL BRAIN IMAGING CENTRE. BIC - The McConnell Brain Imaging Centre: ICBM $152 \mathrm{~N}$ Lin 2009. The McConnell Brain Imaging Centre, 1997. Disponivel

em: $<$ http://www.bic.mni.mcgill.ca/ServicesAtlases/ICBM152NLin2009>. Acesso em: 15 Abril 2015.

SCHMIDT, P.; GASER, C.; ARSIC, M.; BUCK, D.; FÖRSCHLER, A.; BERTHELE, A.; HOSHI, M.; ILG, R.; SCHMID, V. J.; ZIMMER, C. et al. An automated tool for detection of FLAIR-hiperintense white-matter lesions in Multiple Sclerosis. NeuroImage 59, 2012. 3774-3783. 